ISSN 1112-9867

Available online at http://www.jfas.info

\title{
BIOLOGICAL CONTROL BY (COCCINELLA ALGERICA, KOVAR 1977) AGAINST THE PUCERON OF CROPS UNDER GREENHOUSES (STATION BIORESSOURCES OF EL OUTAYA CRSTRA) BISKRA; ALGERIA
}

\author{
M. Rahmouni*1,2, M. Belhamra ${ }^{3}$, M. K. Ben Salah ${ }^{2}$ \\ ${ }^{1}$ Université de Batna 2, faculté de science, Batna ; Algérie \\ ${ }^{2}$ Centre de recherche scientifique et technique sur les régions arides (CRSTRA), \\ Biskra ; Algérie. \\ ${ }^{3}$ Laboratoire diversité des écosystèmes et dynamiques des systèmes de production \\ agricoles en zones arides, université Mohamed Khider. Département des Sciences \\ Agronomiques Biskra; Algérie.
}

Received: 01 June 2017 / Accepted: 26 August 2017 / Published online: 01 September 2017

\begin{abstract}
Inputs from chemicals, particularly pesticides, to control crop pests have adverse effects on soil and the environment, among others. To reduce pest attacks, biological control with indigenous predators is the alternative and the cleanest, most environmentally friendly and ecologically balanced way.

In order to achieve this objective, we carried out ladybird breeding and releases were carried out on vegetable crops under glass in the Bioressources (CRSTRA) station where chemical inputs are not used. This study shows that massive and successive releases larvae Coccinella algerica stage L3 and L4 (10 to 20 larvae C.algerica and $\approx 350$ individuals in Individuals in adult stage / infested plants) reduced effectively the population of aphids.
\end{abstract}

Keywords: Biological control; Coccinella algerica; Vegetable crops; Station Bioressources.

Author Correspondence, e-mail: mimo.crstra@hotmail.fr

doi: http://dx.doi.org/10.4314/jfas.v9i3.21

\section{INTRODUCTION}

En fonction de leur efficacité prédatrice, les coccinelles entomophages ont été classées en prédateurs "de choc" ou de "faiblesse" (Iperti, 1978). A ce titre, la coccinelle de part sa petite 
taille avère se placer dans la deuxième catégorie dans la lutte (Idder, 2011). Aussi, on a estimé utile, d'essayer de quantifier sa consommation en proies. L'étude de la consommation alimentaire des coccinelles prédatrices a fait l'objet de nombreux travaux dans le monde mais dans la plupart des cas, ceux-ci ont été abordés à travers l'effectif théorique de proies éliminées par le prédateur et non la quantité réelle de proies ingérées. En Algérie, la lutte contre les ravageurs de cultures passe généralement par des produits chimiques, coûteux et entraîner des plusieures problèmes par pollution des eaux et des sols qui menace sur la santé humaine (Multigner, 2005) ; développement de résistance chez certains ravageurs et pathogènes; destruction de la faune et de la flore auxiliaire.

Afin de palier à ces inconvénients, plusieurs chercheurs se penchent actuellement sur l'utilisation des moyens de lutte biologique dans le but de limiter les pullulations et la nocivité des diverses ennemis des cultures suite à la disparition des ennemis naturels (Sahraoui, 1998). Cependant, l'utilisation à grande échelle de ces ennemis naturels pose les problèmes de leur production en grande quantité. En ce qui concerne les insectes utiles, un des points délicats est leur alimentation.

Les études menées à ce jour sur l'inventaire et les fluctuations des populations de pucerons dans plusieurs régions d'Algérie, montrent que la situation est très grave et nécessite une intervention urgente (Aroun 1985, 1998 Boughnou et Betam).

La faune auxiliaire constitue l'un des principaux facteurs de limitation des ravageurs et parmi cette faune, Coccinella algerica constitue un groupe entomophage susceptible de jouer un rôle important dans la réduction des populations de puceron. Pour cette raison on a effectué des essais de lutte biologique par élevage et lâcher de cette espèce indigène au niveau des cultures légumières sous-serres dans la station Bioressources d'El'Outaya du (CRSTRA); ou la conduite des cultures est en « bio ».

Dans ce cadre, la lutte biologique en cultures sous-serres vise à fournir aux agriculteurs, des références permettant une utilisation raisonnée et raisonnable.

\subsection{Materiels et methodes}

\subsubsection{Présentation du milieu d'étude}

Un essai est installé à la station Bioressources du C.R.S.T.R.A (N 36 55'36.6”, E 005³8'56"), située dans la plaine d’El'Outaya, au Nord-ouest de la ville de Biskra, fait partie de la zone des Ziban et s'étend sur environ 22 ha. Elle constitue une entité géographique formant une cuvette fermée (Fig.1 et fig. 2). 


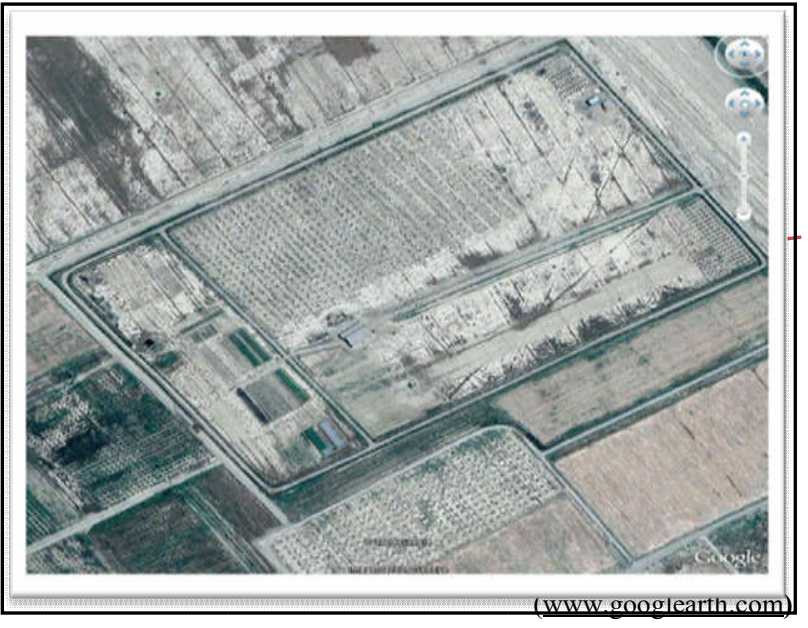

Fig.1. Image satellitaire de la station Bioressources d'El'Outaya.

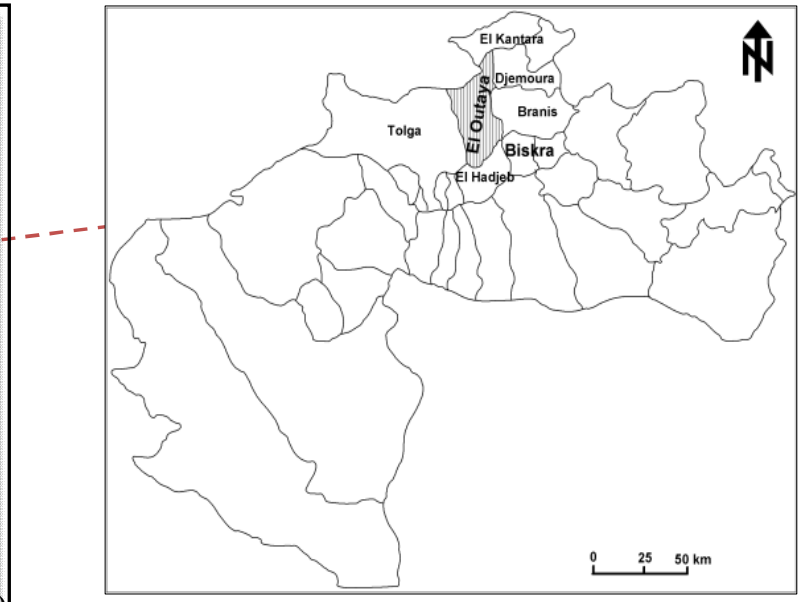

Fig.2. Position géographique de la station Bioressources d'El'Outaya.

\subsubsection{Matériel animal}

\subsubsection{Elevage en masse des Coccinelles}

L'élevage de $C$. algerica est associé à celui de puceron. En effet, ce dernier constitue la ressource alimentaire des larves de coccinelles. Pour cet élevage, nous avons utilisé deux cages grillagées $(70 \mathrm{~cm} \times 60 \mathrm{~cm} \times 50 \mathrm{~cm})$, munies d'une ouverture recouverte d'une toile à mailles de $1 \mathrm{~mm}$ (pour assurer l'aération). Recoit 13 individus de C. algerica sans distinction de sexe, et dont le fond est recouvert de rondelles de papier filtre, de façon à absorber les déjections des coccinelles. Ces supports de papier servent en même temps de substrat de ponte. Ils sont retirés et changés chaque les 24 heures ainsi qu'une réserve d'eau pour garder une humidité $70 \%$.Un chauffage électrique assure une température variant de 25 à $30^{\circ} \mathrm{C}$ et humidité relative moyenne de $70 \%$ (Fig. 3).

Les jeunes larves sont recueillies dans des boîtes en plastique servent d'éclosoirs au moyen d'un pinceau souple, et transférées individuellement à raison d'une larve par boîte. Elle y restera tout le temps de son développement pré-imaginal en présence d'une abondante qantité de pucerons.

\subsubsection{Matériel végétal}

Pour entamer la lutte biologique par Coccinella algerica on a choisi une culture sous-serre de la famille des solanacées représentée par le piment hybride (Capsicum annuum F1) celui-ci est installé le 10 septembre 2014 cette culture est conduite en bio. Un total de 24 plantes de 
piment/serre est utilise pour calculer la moyenne de pucerons par plant et évaluer l'effectif de la population de coccinelle nécessaire pour éliminer les pucerons et limiter les dégâts.

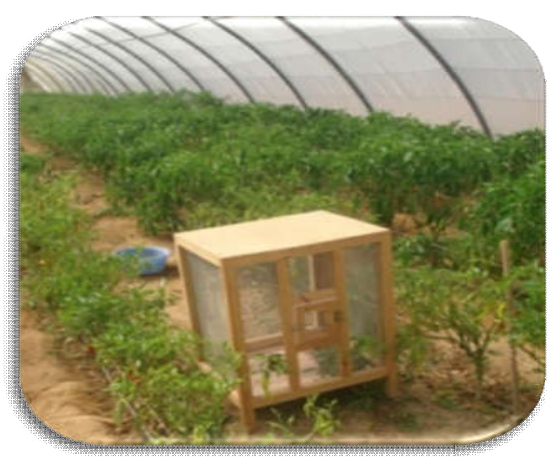

Fig.3. Cage d'élevage au sein de la serre de piment

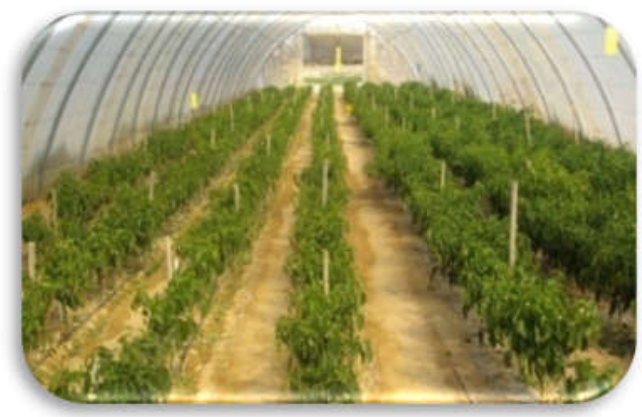

Fig.4. Dispositif de surveillance de puceron

\subsubsection{Méthodes de lâchers}

Deux méthodes d'intervention sont appliquées :

- Lâchers massifs des larves indigènes de C. algerica provenant d'élevage qui être une méthode prometteuse et peut permettre de contrôler les populations de pucerons (Wyss et al. 1999), et renouvèler le lâcher au moment de la nymphose de coccinelle s'il ya des pucerons.

- Lâchers inoculatifs répétés par de quelques individus d'adultes indigènes de C.algerica.

\section{Résultats et discussion}

\subsection{Elevage de C.algerica}

Au cours d'élévage de C.algerica réalisées au sein du laboratoire, nous avons constaté que la voracité augmente surtout dans les stades larvaires L3 et L4; ou la voracité d'une larve de stade trois est de 188 jusqu'au 243 puceron par jour à des temperatures varaint entre 27 et $30^{\circ} \mathrm{C}$. Pour cette raison on a suivi le cycle de vie de $C$. algerica sous les conditions naturelles (sous-serre) et au sein du laboratoire, pour déterminer le nombre efficace de coccinelle à apporter pour éliminer les pucerons et limiter les pertes.

\subsubsection{Cycle de développement au laboratoire}

- Ponte de C.algerica

Les résultats de l'étude sont présentés dans les tableaux suivants sous forme des données comparatives (laboratoire et la serre). 
Tableau 1. Nombre d'œufs pondus par femelle au niveau du laboratoire et dans la serre.

\begin{tabular}{|c|c|c|}
\hline $\begin{array}{c}\text { Les couples de C. algerica } \\
\text { (3 répétitions) }\end{array}$ & $\begin{array}{c}\text { Nombre d'œufs/ponte/femelle } \\
\text { au laboratoire }\end{array}$ & $\begin{array}{c}\text { Nombre d'œufs /ponte/ femelle } \\
\text { dans la serre }\end{array}$ \\
\hline Femelle 1 & 105 & 47 \\
\hline Femelle 2 & 64 & 42 \\
\hline Femelle 3 & 123 & 49.33 \\
\hline Moyenne & 97.33 & \\
\hline
\end{tabular}

Au moment de la ponte, la femelle choisit une feuille envahie de pucerons. Elle s'y installe et commence à pondre ses œufs, au nombre différent selon les conditions écologiques de biotope. La ponte de $C$. algerica varie d'une femelle à une autre de 64 à 123 œufs/femelle pour une température située entre $25^{\circ} \mathrm{C}$ et $30^{\circ} \mathrm{C}$ au niveau du laboratoire par contre dans la serre, la ponte varie de 42 à 59 œufs/femelle pour la température située entre $24.53^{\circ} \mathrm{C}$ et $27.42^{\circ} \mathrm{C}$.

La température et l'alimentation agissent sur la multiplication de la C.algerica.

- Durée des differents stades larvaires de $C$. algerica

Le suivi de la durée des différents stades larvaires dans le laboratoire et dans la serre est présenté dans le tableau 2.

Tableau 2. Durée des différents stades larvaires de C. algerica.

\begin{tabular}{|c|c|c|c|}
\hline \multirow{2}{*}{ Stades larvaires } & \multirow{2}{*}{ Taille $(\mathrm{mm})$} & \multicolumn{2}{|c|}{ Durée moyenne de développement (j) } \\
\cline { 3 - 4 } & & Serre & Laboratoire \\
\hline L1 & 1 & 2 & 2 \\
\hline L2 & 2 à 3 & 6 & 3 \\
\hline L3 & 4 à 6 & 5 & 4 \\
\hline L4 & 7 à 12 & 5 & 11 \\
\hline
\end{tabular}


Le temps d'incubation est sensiblement identique pour les deux conditions (laboratoire et sous-serre) de 3 à 5 jours respectivement à des températures moyennes de $27^{\circ} \mathrm{C}$ et $30^{\circ} \mathrm{C}$.

Les larves de la $C$. algerica ont mué 3 fois, on note 4 stades larvaires, ayant des durées différentes. La durée moyenne de développement de l'état larvaire de $C$. algerica sous les conditions de la serre est de 18 jours pour une température qui varie entre $20^{\circ} \mathrm{C}$ et $27^{\circ} \mathrm{C}$ et une humidité relative de 65 à 70\%. Et dans les conditions contrôlées (laboratoire) la durée moyenne de développement de l'état larvaire est de 11 jours pour de température varie entre $25^{\circ} \mathrm{C}$ et $30^{\circ} \mathrm{C}$ avec une humidité relative de $65 \%$ à $75 \%$.

C'est au niveau des stades larvaires (surtout les L2 et L3) que les différences de durée du développement sont les plus marquées.

Ces valeurs peuvent varier en fonction des paramètres climatiques et des apports alimentaires disponibles. Les larves des coccinelles sont d'une voracité importante, surtout celles du $3^{\text {ème }}$ et $4^{\text {ème }}$ stade larvaire pour assurer leurs croissances.

\section{- Longévité et fécondité des femelles}

Le début de la ponte intervient en moyenne 8 jours après l'émergence de la femelle de C.algerica dans les conditions contrôlées $\left(30^{\circ} \mathrm{C}\right.$ et une humidité relative de $\left.75 \%\right)$; peut être précoce de 2 jours pour les conditions sous-serre $\left(25^{\circ} \mathrm{C}\right.$ et une humidité relative de $\left.65 \%\right)$.

\section{- Période d'activité et de présence de $C$. algerica}

L'activité des coccinelles varie suivant le degré de présence des proies sur les cultures et d'autres facteurs écologiques, comme le micro-climat du biotope fréquenté et les conditions climatiques saisonnières de la région étudiée.

Tableau 3. Périodes d'activité et de présence de $C$. algerica dans le site d'étude

\begin{tabular}{|c|c|c|c|c|c|c|c|c|c|c|c|c|}
\hline Période & $\mathrm{S}$ & $\mathrm{O}$ & $\mathrm{N}$ & $\mathrm{D}$ & $\mathrm{J}$ & $\mathrm{F}$ & $\mathrm{M}$ & A & $\mathrm{M}$ & $\mathrm{JU}$ & JUI & OU \\
\hline $\begin{array}{l}\text { Coccinella } \\
\text { algerica }\end{array}$ & & & & & & & & & & & & \\
\hline
\end{tabular}

$56.3 \%$ Activité intense.

07\% Une très faible densité des adultes.

$0 \%$ Absence (hivernation).

$0.2 \%$ Période de présence sur la culture. 
L'activité de cette coccinelle est un peu précoce par rapport au d'autres auxillaires dans notre site d'étude an raison des bonnes conditions climatiques et de l'abondance de la nourriture, celle-ci a été favorisée par une végétation fraîche et bio; les premiers individus adultes de coccinelles s'installent sur les cultures vers la dernière décade du mois de janvier et la reproduction commence vers le début du mois de Mars (présence des œufs de la première génération).

Dans notre site, nous avons enregistré durant cette période (September 2014 jusqu'au Aout 2015) des minima supérieurs à $8.01^{\circ} \mathrm{C}$ en hiver et des maxima supérieur à $29.47^{\circ} \mathrm{C}$ en été, ces contraintes climatiques vont obliger les coccinelles à arrêter temporairement leur activité reproductrice; péroide de diapause qui s'étale de la mi- juin $\left(29^{\circ} \mathrm{C}\right)$ jusqu'à la première décade du mois de septembre (une absence totale des œufs et des larves et une très faible densité d'adultes). Malgré la présence de la nourriture (puceron), les adultes de $C$. algerica ralentissent leur activité et choisissent souvent les endroits ombragés qui se trouvent soit au niveau des roches ou dans les fissures de la terre pour se protéger des effets du soleil.

L'activité des coccinelles reprend vers la fin du mois de septembre, suite à l'amélioration des conditions climatiques (Température inférieurs à $29^{\circ} \mathrm{C}$ ) et l'installation des populations de puceron vers la fin de septembre. Ainsi, les premières larves de la deuxième génération automnale des coccinelles ont été récoltées vers la fin de septembre, l'activité larvaire s'étale jusqu'à la première quinzaine du mois de novembre.

\subsection{Lutte biologique contre les pucerons de piment sous serre}

Cet essai a pour objectif de tester une méthode de lutte biologique contre le puceron sur le piment sous-serre et déterminer le nombre des individus de $C$. algerica pour lutter contre ce ravageur.

La lutte contre le puceron du piment sous-serre passe tout d'abord par la sélection de variétés résistantes (attirant et abritant peu de pucerons) ou tolérantes (tolérant de fortes densités de pucerons sans en affecter le rendement).

Seuil d'alerte $=250$ pucerons par plant est observé pour $80 \%$ des plants échantillonnés (Roy ,2004) 


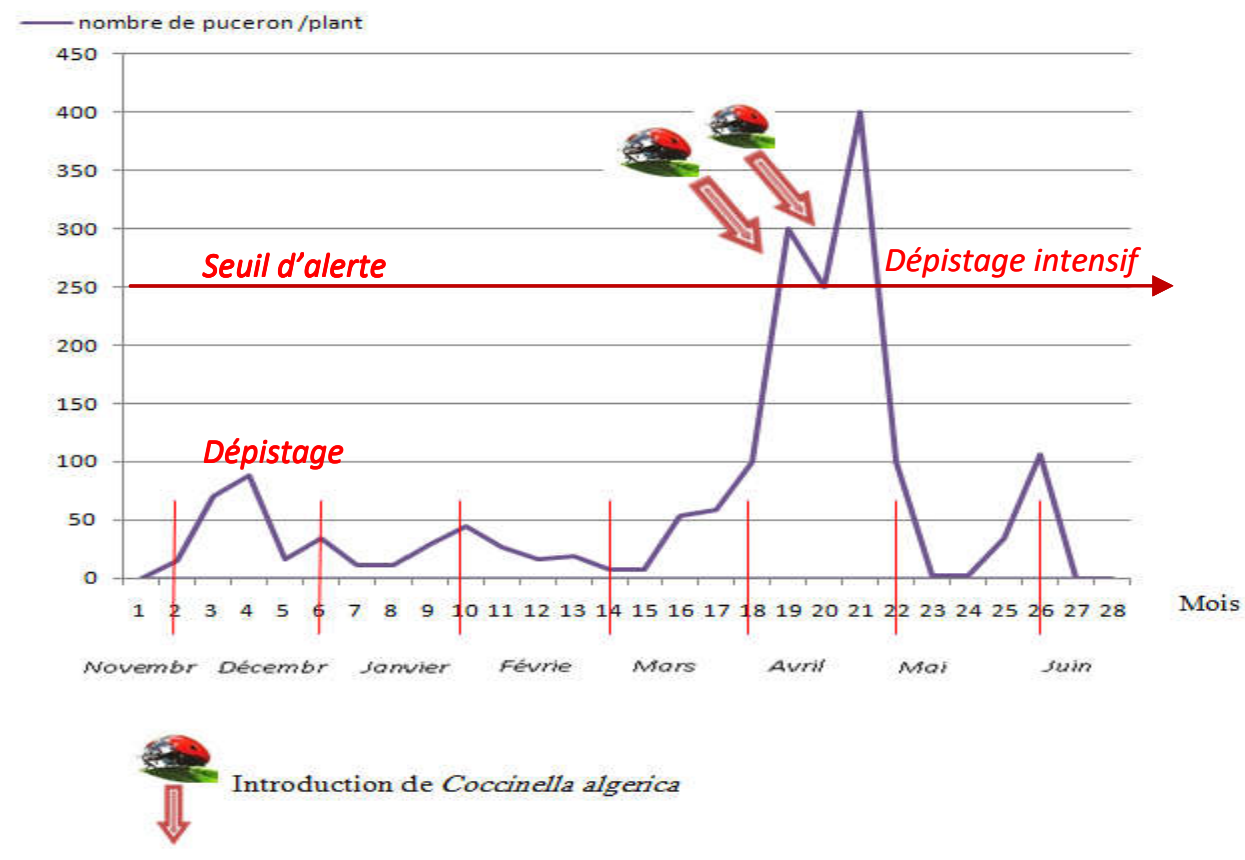

Fig.5. Evolution de population du puceron en fonction de C. algerica

Les premières colonies du Aphis crassivora, Aphis gossypii, Aphis. fabae sont apparues à la fin de novembre ou les populations restent basses jusqu'à la première quinzaine de mois d'avril, avec moins de 250 pucerons pour $80 \%$ des plants (Fig. 5).

À partir de cette densité de pucerons, la serre est à surveiller aux deux ou trois jours après ou les populations demeurent en augmentation après l'atteinte de 338 individus /plant sur une moyenne de 24 plants échantillonnés. Des lachers massifs des larves de C. algerica (10 à 20 larves/plant infesté) sont appliqués.Après cinque jours les effectifs de puceron sont diminuent suite à l'action efficace de cette espèce de coccinelle .La courbe de population des pucerons connaît alors un pallier avant de chuter rapidement.

Par ailleurs, en 2 semaines, le nombre de pucerons est redescendu de nouveau à cause de ralentissement d'activité des larves de coccinelle se transforment en nymphe (la consommation du puceron est nulle), dans cette période on a renouvelé le lâcher de C.algerica et cette fois en stade d'adulte (350 individus de coccinelle) pour une lutte lente etprogressive, on remarque que les populations sont diminuent jusqu'à la fin mai.

Par la suite, les populations de pucerons sont restées très faibles durant la période de l'essai. 
Alors la forte présence de Coccinella algerica a créé un impact direct sur la population des pucerons.

\section{CONCLUSION}

Cet essai a pour objectif de tester une méthode de lutte biologique contre les pucerons des cultures légumières sous-serre présenté par le piment. La forte limitation de traitements phytosanitaires sur la station Bioressources d 'El'Outaya (C.R.ST.R.A) depuis de nombreuses années, a permis la colonisation par un ennemie naturel de puceron qu'est Coccinella algerica.

Les résultats de cette étude montrent que les lâchers massifs de Coccinella algerica peuvent apporter un contrôle suffisant des pucerons qui offre une alternative intéressante aux traitements chimiques. D'une part, le recours aux auxiliaires se révèle efficace, surtout si un suivi régulier est pratiqué. D'autre part, l'absence des traitements chimiques permet aux auxiliaires naturels de s'installer ce qui limite d'autant les besoins de lutte contre les pucerons. Plusieurs conclusions peuvent être tirées de l'essai: Les pucerons majoritairement présents sur le piment sont Aphis crassivora, Aphis gossypii, A. fabae, Aphis frangulae, M. persicae ,Myzacallis castanicola et Aphis nasturtii .

En complément de ces résultats, il est important de rappeler différents points pour s'assurer de l'efficacité du lâcher de Coccinella algerica:

Trois stades phénologiques de piment ont ainsi été pris en considération durant la lutte : repiquage, floraison et récolte.

Les coccinelles doivent être introduites au stade L 3 et L4 (4 -12 mm). Avant, les larves sont trop fragiles et plus tard, le risque de cannibalisme, à cause du manque de nourriture dans les cages d'élevage des coccinelles, devient trop important.

$>$ La période d'activité de puceron en corrélation avec la période d'activité de C.algerica qui s'étale entre le mois de mars jusqu'a la deuxième semaine du mois de juin.

Le lâcher doit être réalisé sur une population de pucerons modérée. Si les populations ont explosé, il est souhaitable préalablement un lâché préventif successif de C.algerica.

Les coccinelles cohabitent et partagent leur nourriture avec d'autres prédateurs et parasitoïdes. Lés aphidiphage comportent sept espèces réparties dans quatre familles: les Coccinellidae, les Syrphidae, les Chrysopidae et les Braconidae. 
L'idée d'intégrer tous ces ennemis naturels dans un vaste programme de lutte intégrée en associant également d'autres méthodes de lutte, entre autres l'aménagement des agrosystèmes, reste la seule alternative pour protéger ces cultures.

\section{REMERCIEMENTS}

A tous le personnel de la station Bioressources, et une mention spéciale doit être faite à messieurs Djoudi Madjed et Bensaleh Med pour leur aide.

\section{REFERENCES BBIBLIOGRAPHIQUES}

[1] Aroun M.E.F., 1985. Les aphides et leurs ennemis naturels en vergers d'agrumes de la Mitidja. Thèse. Magister.Inst.Nat.Agro. El- Harrach, Alger, 125 p.

[2] Alford D.V., 1994. Atlas en couleur. Ravageurs des végétaux d'ornement - arbres, arbustes, fleurs. INRA Éditions. Paris. 92 p.

[3] Blackman R. L., et Eastop V. F., 1984. Aphids on the World's Crops (Avon, British Muséum) 123 p.

[4] Betam A., 1998. Contribution à l'étude des pucerons et leurs ennemis naturels dans la région de Bir Touta (Batna). Thèse .Ing. Agro. Univ. Batna. 82 p.

[5] Boughnou N., 1998. Etude des pucerons et leurs ennemis naturels dans un verger d'oranger dans la région d'Oued Aissi (Tizi Ouzou). Thèse .Ing. Agro. Univ. Tizi-Ouzou. 86 p.

[6] Kamel, M. B. H., Rebhi, R., \& Ommezine, A. (2011). Habitats et proies de Coccinella algerica Kovar dans différentes régions côtières de la Tunisie. Entomologie faunistiqueFaunistic Entomology.

[7] Deguine J. P. et Leclant F., 1997. Aphis gossypii Glover (Hemiptera, Aphididae). Les déprédateurs du cotonnier en Afrique tropicale et dans le reste du monde. Ed. CIRAD. France. $112 \mathrm{p}$.

[8] Florent L., 2006. Etude expérimentale des effets maternels chez Aphis gossypii. U.M.R.R.O.S.E 1112. Sophia Antipolis, Université de Nice - INRA. 27 p.

[9] Fraval A., 2006. Aphis fabae (Hém. Aphididé). La découverte de la parthénogenèse, in « Les pucerons $»$. .Insectes $n^{\circ} 141$. (2). $141 \mathrm{p}$.

[10] GEORCRET et SCHEROMM, 1995. Lutte contre les insectes ravageurs des cultures : les apports de la biologie. Ed.INRA, France. 42 p. 
[11] Giordanengo Ph., Febvay G., Rahbé Y., 2007 .Comment les pucerons manipulent les plantes. BIOFUTUR 279. EA3900 Biologie des plantes et contrôle des insectes ravageurs, université de Picardie Jules Vernes 38 p.

[12] Hullé M. et Turpeau-Aït Ighil E., Robert. T.M. et Monnet Y., 1999. Les pucerons des plantes maraîchères. Cycles biologiques et activités de vol. ACTA/INRA Éditions. Paris. 136 p.

[13] Iperti G., 1965.Contribution à l'étude de la capacité chez les principales aphidiphages des Alpes-Maritime et Basses- Alpes.Entomophaga .10: 178 p.

[14] Iperti G., 1978.Influence des principaux facteurs du climat sur le comportement de voi d'une coccinelle aphidiphage Semiadalia undecimnotata schn . ann .zool-ecol .10 : 393 p.

[15] IDDER, A. (2011). Lutte biologique en palmeraies algériennes (Doctoral dissertation).

[16] Jerry M. et Gouzé J.L., 2004.Stratégie optimale d'un problème dans le cadre de la lutte biologique $\mathrm{N}^{\circ}$ 5253. $34 \mathrm{p}$.

[17] Labonne G., Fauvel C., Leclant F., Quiot J. B., 1982. Description d'un piège à succion : son emploi dans la recherche des aphides vecteurs de virus transmis sur le mode non persistant. Agronomie. 2 (8).776 p

[18] Leclant F., 1978. Etude bioécologique des aphides de la région méditerranéenne. Implications agronomiques. Thèse Doctorat es Science. Université de Montpellier, 318 p.

[19] Leclant F., 1982. Les effets nuisibles des pucerons sur cultures. ACTA, Paris, 57 p.

[20] Leclant, F. 2000. Les pucerons des plantes cultivées. Clefs d'identification. III - Cultures fruitières. ACTA et INRA. $128 \mathrm{p}$.

[21] Loussert R. ,1989. Les agrumes 2. Production. Ed. Scientifique universitaire, 113 p.

[22] Multigner, L. (2005). Effets retardés des pesticides sur la santé humaine. Environnement, Risques \& Santé, 4(3), 187-194.

[23] Pfiffner L.,Luka H., Schlatter C., 2005., Funktionelle Biodiversität, Schädlingsregulation gezielt verbessern [Functional biodiversity, targeted regulation of pests]. Ökologie und Landbau $134.53 \mathrm{p}$.

[24] Kamel, M. B. H., Rebhi, R., \& Ommezine, A. (2011). Habitats et proies de Coccinella algerica Kovar dans différentes régions côtières de la Tunisie. Entomologie faunistiqueFaunistic Entomology.

[25] Rebhi R., 2008. Bioécologie de Coccinella algerica Kovàr (Coleoptera, Coccinellidae). Mastère en protection des plantes et environnement. ISA Chott Mariem,.Tunisie.95 p. 
[26] Rebhi R. et Ben Halima K. M., (2009). Bioécologie de Coccinella algerica dans une région côtière de la Tunisie. Actes du congrès international sur la diversité biologique des invertébrés en milieux agricoles et forestiers. INA El Harrach. Alger 14- 17 Avril 2008. 61p.

[27] Sforza R. 2009. Utilisation d'organismes phytophages. In Pintureau, B. (réd.), La lutte biologique : Application aux arthropodes ravageurs et aux adventices (Chapitre VII. Paris, Ellipses Éditions. 145 p.

[28] Saharaoui 1. et Gourreau J.M ., 2000. Etude de quelques paramètres bioécologiques des coccinelles aphidophages d'algérie (coleoptera, coccinellidae).18 p.

[29] Saharaoui L., Iperti G. et Gourreau J.M. 2001. Les coccinelles d'Algérie: inventaire préliminaire et régime alimentaire (Coleoptera, Coccinellidae). Bulletin de la Société Entomologique de France 103(3), p. 216-219

[30] Skinner B. et Domaine É., 2010 .rapport sur la situation de la coccinelle à deux points (Adalia bipunctata) au Québec .Ministère des Ressources naturelles et de la Faune Québec. $29 \mathrm{p}$.

[31] Taylor L.R., Palmer J.M.P., Dupuch M.J., Cole J., et Taylor M.S., 1981. A handbook for the rapid identification of alate aphids of Great Britain and Europe. Rothamsted Exp.Sta., Harpenden. 171 p.

[32] Vaissayre M. et Cauquil J. 2000. Principaux ravageurs et maladies du cotonnier en Afrique au nord du Sahara .CIRAD ICTA .60 p.

[33] Wyss E., Villiger M., Hemptinne J.L. \& Muller-Scharer H. (1999). Effects of augmentative releasesof eggs and larvae of the ladybird beetle, Adaliabipunctata, on the abundance of the rosy apple aphid, Dysaphis plantaginea, in organic appleorchards.

Entomologia Experimentalis et Applicata 90, p. 167-173.

\section{RESUME}

Les apports des produits chimiques et notamment les pesticides pour la lutte contres les déprédateurs des cultures sous serres ont des effets néfastes sur l'environnement. Pour réduire les attaques des bioagressuers des cultures, la lutte biologique avec les prédateurs autochtones constitue l'alternative et le moyen le plus avantageux et le plus respectueux de l'environnement.

Pour atteindre cet objectif un élevage de Coccinella algerica est mené, et des lâchers ont été effectués sur cultures légumières sous serre où les intrants chimiques ne sont utilisés. Les 
lâchers massifs et successif des larves de Coccinella algerica au stade L3 et L4 (10 à 20 larves de C.algerica et 350 individus adultes/plants infestés) ont réduit d'une manière efficace la population des pucerons.

Mots clefs: Lutte biologique ; Coccinella algerica; pucerons ; cultures légumières.

\section{How to cite this article:}

Rahmouni M, Belhamra M, Ben Salah MK.Biological control by (Coccinella algerica, kovar 1977) against the puceron of crops under greenhouses (station bioressources of el outaya crstra) biskra; Algeria. J. Fundam. Appl. Sci., 2017, 9(3), 1585-1597. 